\title{
GMR
}

\section{Hypoxia-induced apoptosis and mitochondrial dysfunction in chondrocytes arising from CREB phosphorylation reduction}

\author{
Y.Y. Qiu, Y. Chen, T.H. Zeng, W.H. Guo, W.Y. Zhou and X.J. Yang \\ Department of Spine Surgery, The 2nd Shenzhen People's Hospital, \\ Shenzhen, China \\ Corresponding author: X.J. Yang \\ E-mail: jian_xinyang@163.com \\ Genet. Mol. Res. 15 (2): gmr.15027755 \\ Received December 16, 2015 \\ Accepted January 15, 2016 \\ Published June 10, 2016 \\ DOI http://dx.doi.org/10.4238/gmr.15027755
}

\begin{abstract}
Chondrocytes, which are embedded within the growthplate or the intervertebral disc, are sensitive to environmental stresses, such as inflammation and hypoxia. However, little is known about the molecular signaling pathways underlying hypoxia-induced mitochondrial dysfunction and apoptosis in chondrocytes. We first examined the apoptosis, caspase-3 activity, and apoptosis-associated markers in human chondrocyte cell line C28/I2 under normoxia or hypoxia. We then investigated mitochondrial dysfunction and the activation of cyclic adenosine monophosphate response elementbinding protein (CREB) signaling in the same human chondrocyte cell line. Our results indicated that hypoxia induced apoptosis and reduced CREB phosphorylation in chondrocytes. Upregulated mitochondrial superoxide and reactive oxygen species levels, and reduced mitochondrial membrane potential and complex IV activity were observed in hypoxia-treated C28/I2 cells. In conclusion, the present study confirmed reduced CREB phosphorylation, apoptosis induction, and mitochondrial dysfunction in the hypoxia-treated chondrocyte
\end{abstract}


cells. This implies the key role played by CREB signaling in hypoxiainduced mitochondrial dysfunction and apoptosis in chondrocytes.

Key words: Hypoxia; Apoptosis; Mitochondrial dysfunction; Chondrocytes; CREB phosphorylation

\section{INTRODUCTION}

Chondrocytes, which are embedded within the growth-plate or the intervertebral disc, survive in an almost avascular and hypoxic milieu, and are sensitive to environmental and multiple cellular stresses, such as inflammation, endoplasmic reticulum(ER) stress and hypoxia (Horton et al., 2006; Chang et al., 2014). Hypertrophic chondrocytes undergo programmed cell death (apoptosis) during cervical spondylosis or joint disorders involving osteoarthritis (OA) characterized by progressive breakdown of articular cartilage (Triantafillou et al., 2012; Sun et al., 2013). Reactive oxygen species (ROS) generated by hypoxia/ischemia trigger apoptosis (Misra et al., 2009).

Mitochondria are the key targets of ROS, which are thought to regulate mitochondrial dysfunction and the promotion of apoptosis. Elevated ROS production causes mitochondrial damage, such as collapse of the mitochondrial membrane potential, and complex IV inactivation, which opens up the mitochondrial permeability transition pores, leading to the release of pro apoptotic proteins into the cytoplasm (Balaban et al., 2005; Choi, 2012). This causes the release of cytochrome $\mathrm{c}$. Cytochrome $\mathrm{c}$ initiates the mitochondria-dependent caspase-3 pathway, which plays a pivotal role in apoptosis (Kroemer et al., 1997; Reed, 1997). ROS also cause structural and functional damage to mitochondria (Chomyn and Attardi, 2003). However, little is known about the molecular signaling pathways underlying the mitochondriadependent caspase pathway.

Cyclic adenosine monophosphate (cAMP) response element-binding protein (CREB) is necessary for cell proliferation and apoptosis (Wang et al., 2013) because it regulates the expression of a repertoire of genes associated with cell survival. Those genes include B-cell lymphoma 2 (BCL2), B-cell lymphoma-extra large (BCLXL), and CFOS (Ferron et al., 2011; Han et al., 2013), and are particularly relevant during hypoxia (Meng et al., 2012). The inhibition of CREB-Ser133 phosphorylation results in the suppression of anti-apoptotic genes (Aggarwal et al., 2008; Naqvi et al., 2014). Therefore, CREB signaling might be implicated in hypoxia-induced apoptosis in chondrocytes.

In this study, we first examined hypoxia-induced apoptosis in human chondrocyte cell line $\mathrm{C} 28 / \mathrm{I} 2$. We then investigated the activation of CREB and mitochondrial dysfunction in hypoxia-promoted apoptosis in the same cell line. Our results implied the involvement of CREB signaling and mitochondrial dysfunction in the hypoxia-induced apoptosis of chondrocytes.

\section{MATERIAL AND METHODS}

\section{Cell culture and treatments}

Human chondrocyte C28/I2cells were purchased from the American Type Culture Collection (ATCC) and were cultivated in Dulbecco's modified Eagle's medium (DMEM) 
(Gibco, Rockville, MD, USA) containing 10\% fetal bovine serum (FBS) (Invitrogen, Carlsbad, CA, USA), 100 units $/ \mathrm{mL}$ penicillin, and $100 \mu \mathrm{g} / \mathrm{mL}$ streptomycin (Sigma-Aldrich, St. Louis, $\mathrm{MO}, \mathrm{USA}$ ) under $5 \% \mathrm{CO}_{2}$ at $37^{\circ} \mathrm{C}$. The cells were sub-cultured after reaching approximately $90 \%$ confluence. For hypoxia treatment, the cells were placed in a hypoxia incubator with $5 \% \mathrm{CO}_{2}$ and $3 \%$ oxygen. Oxygen concentration was monitored continuously (Forma 3130, Thermo Scientific, Rockford, IL, USA).

\section{MTT assay for cellular viability}

The cellular viability of the C28/I2 cells was assessed using a3-(4,5-dimethylthiazol2-yl)-2,5-diphenyl tetrazolium bromide (MTT) assay kit (Invitrogen, Carlsbad, CA, USA). In brief, $\mathrm{C} 28 / \mathrm{I} 2$ cells, post treatment, were mixed with the MTT reagent and incubated at $37^{\circ} \mathrm{C}$ for $4 \mathrm{~h}$, then treated with dimethyl sulfoxide (DMSO) to dissolve the formazan crystals. Optical densities (ODs) were measured at $450 \mathrm{~nm}$ using aspectrophotometer (Bio-Rad, Hercules, CA, USA). Cellular viabilities are presented as average $\mathrm{OD}_{450}$ values.

\section{Apoptosis assay and caspase- 3 assay}

Apoptosis induction inC28/I2 cells was investigated using an annex in V/fluorescein isothiocyanate (FITC) apoptosis detection kit (Abcam, Cambridge, UK). In brief, 1x 106 post treatmentC28/I2cells were stained with an nexin V-FITC and propidium iodide, and examined using a FACS can flow cytometer (BD Biosciences, San Jose, CA, USA). Apoptosis was evaluated by the percentage of an nexin V-positive cells to total cells.Caspase-3 activity in the C28/I2 cells was determined using a Caspase Senso Lyte kit (for caspase-3) (Ana Spec, Fremont, CA, USA). The cells were collected and washed three times with ice-cold phosphate-buffered saline (PBS), then resuspended in $100 \mu \mathrm{L}$ (final volume) of a caspase buffer solution supplemented with the fluorogenic peptide substrate Ac-DEVD-AMC for incubation for $30 \mathrm{~min}$ at $37^{\circ} \mathrm{C}$. The caspase- 3 activity was determined by assessment of AspGlu-Val-Asp (DEVD)-AMC cleavage in a fluorescence spectrophotometer (Hitachi, Tokyo, Japan) with an excitation wavelength of $390 \mathrm{~nm}$ and an emission wavelength of $460 \mathrm{~nm}$. The activity was expressed as percent value of fluorescence intensity of AMC compared with the control.

\section{Western blotting assay}

The mitochondrial and cytosolic proteins in C28/I2 cells were isolated using a Mitochondria/Cytosol Fractionation Kit (Abcam, Cambridge, UK), and were supplemented with a protease inhibitor cocktail (Roche). The protein samples were then separated using $12 \%$ sodium dodecyl sulfate polyacrylamide gel electrophoresis (SDS-PAGE) gels, and were electro-transferred onto nitrocellulose membranes (Millipore). The membranes were incubated with rabbit antibody against cytochrome c (Cytc), caspase-3 (CASP 3), CREB, CREB with Ser133 phosphorylation (all Santa Cruz Biotechnology, Santa Cruz, CA, USA), or $\beta$-actin (Sigma-Aldrich, St. Louis, MO, USA). The specific binding signal was then acquired using ECL detection systems by incubation with horseradish peroxidase-conjugated secondary antibodies (Pierce, Rockford, IL, USA). 


\section{Measurement of mitochondrial superoxide and ROS}

The mitochondrial superoxide was examined using MitoSOX ${ }^{\mathrm{TM}}$ Red mitochondrial superoxide indicator (Invitrogen, Carlsbad, CA, USA) $(\mathrm{Ex} / \mathrm{Em}=510 / 580 \mathrm{~nm})$, which detects the superoxide in mitochondria with highly selectivity. The C28/I2 cells were incubated with $5 \mu \mathrm{M}$ MitoSOX ${ }^{\mathrm{TM}} \mathrm{Red}$ at $37^{\circ} \mathrm{C}$ for $30 \mathrm{~min}$, then detected after the removal of the reagent and washing three times. The level of intracellular ROS was quantified using ROS-sensitive fluorophore 5-(and-6)-chloromethyl-2,7-dichlorodi-hydrofluorescein diacetate (Invitrogen, Carlsbad, CA, USA) according to the manufacturer instructions. Briefly, confluent C28/I2 cells were incubated with the 5-chloromethyl-2,7-dichlorodihydrofluorescein diacetate probe at $37^{\circ} \mathrm{C}$ for $30 \mathrm{~min}$ and washed three times with PBS. The cells were then rinsed with Hank's balanced salt solution (HBSS) and investigated using a fluorescence spectrophotometer (Hitachi, Tokyo, Japan) with an excitation wavelength of $488 \mathrm{~nm}$ and an emission wavelength of $530 \mathrm{~nm}$. The results are reported as a percentage of the control level.

\section{Measurement of mitochondrial membrane potential and complex IV activity}

A TMRE-Mitochondrial Membrane Potential Assay Kit (Abcam, Cambridge, UK) was used to determine the mitochondrial membrane potential (MMP) in the C28/I2 cells. C28/ I 2 cells $\left(3 \times 10^{5}\right)$ were incubated with the MMP-sensitive fluorescent TMRE for 30 minutes at $37^{\circ} \mathrm{C}$ (1000 $\mathrm{nM} \mathrm{FCCP}$ was added to the positive control cells 10 min prior of TMRE). Cells were then trypsinized, centrifuged, and resuspended in $0.4 \mathrm{~mL}$ Dulbecco's phosphatebuffered saline with $0.2 \%$ bovine serum albumin and analyzed for TMRE fluorescence using the fluorescence spectrophotometer (Hitachi, Tokyo, Japan) with an excitation wavelength of $575 \mathrm{~nm}$ and an emission wavelength of $549 \mathrm{~nm}$. Complex IV activity was measured using a Complex IV Rodent Enzyme Activity Microplate Assay Kit (Abcam, Cambridge, UK) according to the manufacturer instructions. In brief, C28/I2 cells were collected and lysed with the extraction detergent. The protein samples were then serially diluted and incubated at room temperature for 3 hon the plate, in each well of which the enzyme-linked monoclonal antibody against complex IV has immobilized. The binding was then assayed using the fluorescence spectrophotometer (Hitachi, Tokyo, Japan) at an excitation wavelength of $575 \mathrm{~nm}$ and an emission wavelength of $549 \mathrm{~nm}$. The results are reported as a percent level of the control.

\section{Statistical analysis}

Statistical analyses were performed using SPSS18.0 software (IBM SPSS). All data are reported as means \pm standard error of the mean. The Student $t$-test was performed to determine the differences between two groups. A P value $<0.05$ was considered statistically significant.

\section{RESULTS}

\section{Hypoxia induces apoptosis in chondrocytes}

We investigated apoptosis induction by hypoxia in chondrocytes by examining cellular viability using the MTT assay. We investigated apoptosis induction using flow 
cytometric analysis, and caspase-3 activity using a Caspase SensoLyte kit (for caspase-3) in the chondrocyte C28/I2 cells under hypoxia or normoxia. The MTT assay results (Figure 1A) demonstrated that the hypoxia treatment for 24 or $48 \mathrm{~h}$ significantly reduced the viability of $\mathrm{C} 28 / \mathrm{I} 2$ cells $(\mathrm{P}<0.05$ or $\mathrm{P}<0.01)$. The apoptosis level was also significantly higher in the hypoxia-treated $\mathrm{C} 28 / \mathrm{I} 2$ cells compared with the C28/I2 cells under normoxia (Figure 1B, P $<0.01$ or $\mathrm{P}<0.001)$. The activity of active caspase-3, revealed by the AMC fluorescence intensity in the hypoxia-treated C28/I2 cells, was also significantly higher in the hypoxia group (Figure $1 \mathrm{C}$ ) at 12,24 , or $48 \mathrm{~h}$ post treatment (H.P.T.) $(\mathrm{P}<0.01$ or $\mathrm{P}<0.001)$.

\section{A}

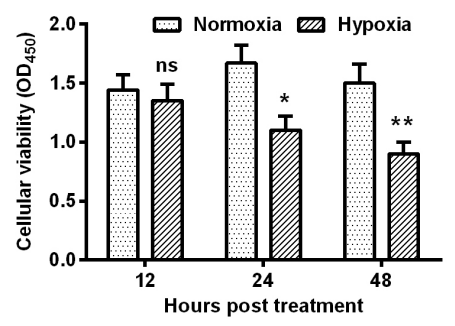

B

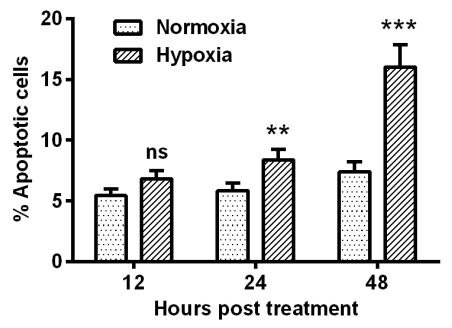

C

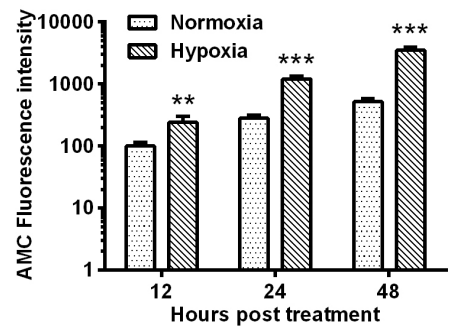

Figure 1. Cellular viability and apoptosis in the hypoxia-treated chondrocyte C28/I2 cells. C28/I2 cells were inoculated under hypoxia or normoxia for 12,24 , or $48 \mathrm{~h}$, and were analyzed for cellular viability using a 3-(4,5-dimethylthiazol-2-yl)-2,5-diphenyltetrazolium bromide (MTT) assay for apoptosis using the an nexin V/ FITC apoptosis detection kit, and for caspase-3 activity with an AMC Caspase-3 Assay Kit. MTT assay results (A), percentage of apoptotic cells (B), and percentage of caspase-3 activity (C) of the C28/I2 cells under hypoxia or normoxia are presented, respectively. Each result was averaged for triple independent experiments. Statistical significance is shown as $* \mathrm{P}<0.05, * * \mathrm{P}<0.01$, or $* * * \mathrm{P}<0.001$; ns $=$ no significance. 


\section{Hypoxia promotes apoptosis-associated markers and CREB phosphorylation in chondrocytes}

To confirm the hypoxia-induced apoptosis in C28/I2 cells, we then analyzed the levels of cytochrome c (Cyt c) released from mitochondria, and activated caspase-3 (cleaved caspase-3, Clv-CASP 3), using western blotting to assay the hypoxia- or normoxia-treated C28/I2 cells. As indicated in Figure 2A, significantly higher levels of Cyt $\mathrm{c}$ were released in the hypoxia-treated $\mathrm{C} 28 / \mathrm{I} 2$ cells at 12,24 , or $48 \mathrm{~h}$ post treatment $(\mathrm{P}<0.05, \mathrm{P}<0.01$, or $\mathrm{P}<$ 0.001 ). There was a time-dependent upregulation of Cyt $\mathrm{c}$ release, with a significant difference between the C28/I2 cells after treatment for 24 and $48 \mathrm{~h}(\mathrm{P}<0.01$, Column 3 vs Column 4 , Figure 2B). Clv-CASP 3 was also markedly promoted by hypoxia treatment after 12, 24, or $48 \mathrm{~h}(\mathrm{P}<0.01, \mathrm{P}<0.001$, or $\mathrm{P}<0.0001$, Figure $2 \mathrm{C})$, also with a time-dependence $(\mathrm{P}<0.05$, Column 3 vs Column 4, Figure 2C).
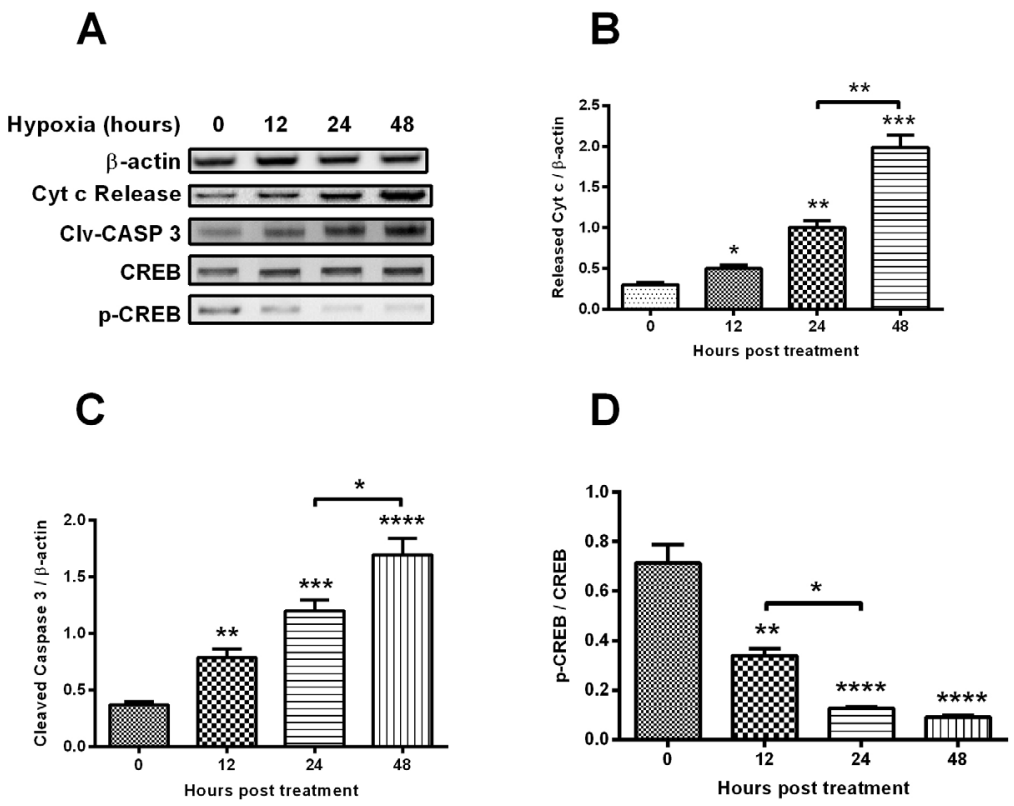

Figure 2. Western blotting analysis of apoptosis-associated markers and CREB phosphorylation in the hypoxiatreated C28/I2 cells. C28/I2 cells were inoculated under hypoxia or normoxia for 12,24 , or $48 \mathrm{~h}$, and a western blotting assay was performed to investigate cytochrome release, cleaved caspase-3, and CREB phosphorylation. A. Representative western blotting results of released cytochrome c (Cyt c release), cleaved caspase-3 (Clv-CASP 3), CREB with (p-CREB) or without phosphorylation (CREB) in the hypoxia-treated C28/I2 cells for $0,12,24$, or 48 h. The level of Cyt c release (B), Clv-CASP 3 (C) and phosphorylated CREB (D) is presented relative to $\beta$-actin. Each result was averaged for triple independent experiments. Statistical significance is shown as $* \mathrm{P}<0.05, * * \mathrm{P}<$ 0.01 , or $* * * \mathrm{P}<0.001$; ns: no significance.

It is well known that CREB facilitates pro-survival signaling (Dworkin et al., 2009), and our study investigated the activation of CREB signaling in hypoxia-treated C28/I2 cells. The western blotting assay indicated that phosphorylated CREB was more markedly downregulated in hypoxia-treated C28/I2 cells than in normoxia-treated C28/I2 cells, whereas 
CREB without phosphorylation was not markedly regulated by hypoxia in $\mathrm{C} 28 / \mathrm{I} 2$ cells $(\mathrm{P}<$ 0.01 or $\mathrm{P}<0.0001$, Figure $2 \mathrm{~A}$ and $2 \mathrm{D})$, time-dependently $(\mathrm{P}<0.01$, Column 2 vs Column 3 , Figure 2D). We confirmed apoptosis induction by hypoxia in chondrocyte C28/I2 cells with the downregulation of CREB phosphorylation.

\section{Hypoxia induces mitochondrial dysfunction in chondrocyte C28/I2 cells}

We then investigated whether hypoxia induced chondrocyte mitochondrial dysfunction. First, we examined superoxide generation using MitoSOX, a live-cell-permeable and mitochondrial localizing superoxide indicator, in C28/I2 cells under hypoxia or normoxia. As shown in Figure 3A, there was a significantly high level of superoxide in the hypoxia-treated cells at both 24 and $48 \mathrm{~h}$ post treatment $(\mathrm{P}<0.01$ or $\mathrm{P}<0.001)$. Second, we measured ROS production in the hypoxia-treated C28/I2 cells. Figure 3B demonstrates that hypoxia enhanced ROS production from 12 to $48 \mathrm{~h}$ post treatment $(\mathrm{P}<0.05, \mathrm{P}<0.01$, or $\mathrm{P}<0.001)$. However, MMP, a well-confirmed indicator of mitochondrial function, was significantly reduced in the hypoxia-treated $\mathrm{C} 28 / \mathrm{I} 2$ cells at 24 or $48 \mathrm{~h}$ post treatment $(\mathrm{P}<0.05$ for 24 H.P.T. or $\mathrm{P}<0.01$ for 48 H.P.T.) (Figure 3C). The activity of mitochondrial respiratory chain complex IV was markedly downregulated in the hypoxia-treated C28/I2 cells (Figure 3D), at 12, 24, or $48 \mathrm{~h}$ post treatment $(\mathrm{P}<0.05, \mathrm{P}<0.01$, or $\mathrm{P}<0.001)$. Therefore, hypoxia promotes mitochondrial dysfunction in chondrocyte $\mathrm{C} 28 / \mathrm{I} 2$ cells.

A

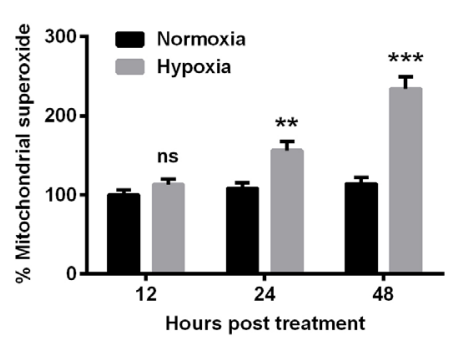

C

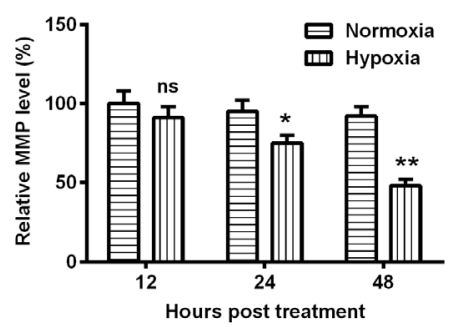

B

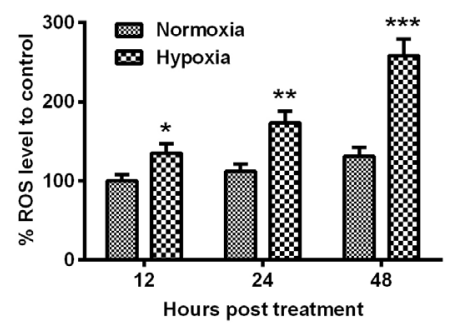

D

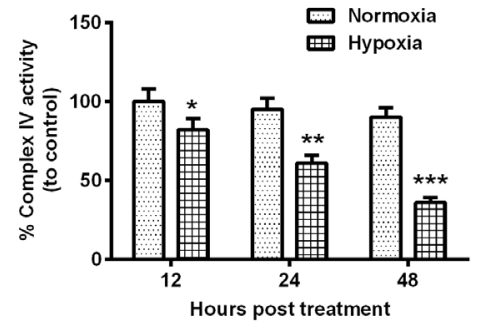

Figure 3. Mitochondrial dysfunction in the hypoxia-treated C28/I2 cells. C28/I2 cells were inoculated under hypoxia or normoxia for 12,24 , or $48 \mathrm{~h}$, and the percentage of mitochondrial superoxide levels (A), reactive oxygen species (ROS) levels (B), mitochondrial membrane potential (MMP) (C), and complex IV activity (D) were assayed. All results were averaged for three independent experiments. ${ }^{*} \mathrm{P}<0.05,{ }^{*} \mathrm{P}<0.01, * * * \mathrm{P}<0.001$; ns: no significance. 


\section{DISCUSSION}

Oxygen affects the activity of many types of cell and is involved in many processes that are important for degenerative bone disease (White and Gibson, 2010a; Dalle Carbonare et al., 2015; Vanharanta, 2015). In this study, we found that hypoxia promoted apoptosis in chondrocyteC28/I2 cells by upregulating caspase-3 activity and inducing mitochondrial dysfunction. Apoptosis can be initiated through membrane receptor-associated and mitochondrial-initiated pathways that converge and mediate downstream apoptosis effects (Hen Gartner, 2000). Mitochondrial dependence was confirmed in the hypoxiainduced apoptosis ofC28/I2 cells. Mitochondrial dysfunction, such as the overproduction of mitochondrial superoxide and ROS, is one of the most important mechanisms in the response to hypoxia in chondrocytes (Milner et al., 2007; White and Gibson, 2010a; Lane et al., 2015). However, hypoxia inhibits complexes I, III, and IV (White and Gibson, 2010b), and MMP (Clérigues et al., 2013) in chondrocytes. Our study confirmed the marked promotion of mitochondrial superoxide and ROS, and the inhibition of MMP and complex IV in hypoxia-treated C28/I2 cells.

Oxidative stress has been implicated in the pathogenesis of osteoporosis. It is conceivable that mitochondrial-mediated ROS generation under oxidative stress leads to the damage of ROS-producing cells and peripheral chondrocytes. As major sources of ROS production, mitochondria are also major targets for ROS attack. The present study demonstrated that oxidative stress mimicked by hydrogen peroxide significantly exerted mitochondrial dysfunction in $\mathrm{C} 28 / \mathrm{I} 2$ chondrocyte cells. The anti-oxidative stress effects exerted by allicin have been identified in ischemic/reperfused rats by inhibiting the activation of JNK-1 and C-JUN and further inhibiting apoptosis (Sato et al., 2001). However, the present study indicated that allicin inhibits ROS generation and ameliorates ROS-induced mitochondrial dysfunction. In addition, allicin treatment ameliorated the repression of PI3K/AKT and CREB/ERK signaling by hydrogen peroxide, and might also be associated with the anti-oxidative stress effect of allicin. Previously, PI3K and CREB have been implicated in chondrocyte-like cell proliferation and differentiation (Carpio et al., 2001; Matsuo et al., 2006). In the present study, allicin treatment of C28/I2 cells increased PI3K activity, and the phosphorylation of AKT, CREB, and ERK. PI3K is a lipid kinase and generates phosphatidylinositol-3,4,5-trisphosphate, which directly or indirectly affects CREB phosphorylation (Vivanco and Sawyers, 2002). CREB is a ubiquitous transcription factor in the higher eukaryotes that, once phosphorylated, promotes the synthesis of mitochondrially encoded subunits of oxidative phosphorylation complexes (De Rasmo et al., 2009). However, since the PI3K/AKT and CREB/ERK signaling pathways are suppressed by hydrogen peroxide, the attenuation of repressed PI3K and CREB by allicin might be caused by reduced hydrogen peroxide toxicity. Moreover, the attenuation of PI3K and CREB repression might ameliorate mitochondrial function by regulating the expression of B-cell lymphoma-2 (Bcl-2) and Bcl-extra large (Bcl-xL) (Boucher et al., 2000), which are located in the outer membrane of the mitochondria and regulate mitochondrial function (Chan and $\mathrm{Yu}, 2004)$.

In conclusion, the present study confirmed reduced CREB phosphorylation, and apoptosis induction and mitochondrial dysfunction in hypoxia-treated chondrocyte cells. This implies the key role of CREB signaling in hypoxia-induced mitochondrial dysfunction and apoptosis in chondrocytes. 


\title{
Conflicts of interest
}

\author{
The authors declare no conflict of interest.
}

\section{REFERENCES}

Aggarwal S, Kim SW, Ryu SH, Chung WC, et al. (2008). Growth suppression of lung cancer cells by targeting cyclic AMP response element-binding protein. Cancer Res. 68: 981-988. http://dx.doi.org/10.1158/0008-5472.CAN-06$\underline{0249}$

Balaban RS, Nemoto S and Finkel T (2005). Mitochondria, oxidants, and aging. Cell 120: 483-495. http://dx.doi. org/10.1016/j.cell.2005.02.001

Boucher MJ, Morisset J, Vachon PH, Reed JC, et al. (2000). MEK/ERK signaling pathway regulates the expression of Bcl-2, Bcl-X(L), and Mcl-1 and promotes survival of human pancreatic cancer cells. J. Cell. Biochem. 79: 355-369. http://dx.doi.org/10.1002/1097-4644(20001201)79:3<355::AID-JCB20>3.0.CO;2-0

Carpio L, Gladu J, Goltzman D and Rabbani SA (2001). Induction of osteoblast differentiation indexes by PTHrP in MG63 cells involves multiple signaling pathways. Am. J. Physiol. Endocrinol. Metab. 281: E489-E499.

Chan SL and Yu VC (2004). Proteins of the bcl-2 family in apoptosis signalling: from mechanistic insights to therapeutic opportunities. Clin. Exp. Pharmacol. Physiol. 31: 119-128. http://dx.doi.org/10.1111/j.1440-1681.2004.03975.x

Chang Z, Huo L, Wu Y and Zhang P (2014). HIF-1 a had pivotal effects on downregulation of miR-210 decreasing viability and inducing apoptosis in hypoxic chondrocytes. ScientificWorldJournal 2014: 876363. http://dx.doi. org/10.1155/2014/876363

Choi EM (2012). Magnolol protects osteoblastic MC3T3-E1 cells against antimycin A-induced cytotoxicity through activation of mitochondrial function. Inflammation 35: 1204-1212. http://dx.doi.org/10.1007/s10753-012-9430-0

Chomyn A and Attardi G (2003). MtDNA mutations in aging and apoptosis. Biochem. Biophys. Res. Commun. 304: 519529. http://dx.doi.org/10.1016/S0006-291X(03)00625-9

Clérigues V, Murphy CL, Guillén MI and Alcaraz MJ (2013). Haem oxygenase-1 induction reverses the actions of interleukin-1b on hypoxia-inducible transcription factors and human chondrocyte metabolism in hypoxia. Clin. Sci. 125: 99-108. http://dx.doi.org/10.1042/CS20120491

Dalle Carbonare L, Matte' A, Valenti MT, Siciliano A, et al. (2015). Hypoxia-reperfusion affects osteogenic lineage and promotes sickle cell bone disease. Blood 126: 2320-2328. http://dx.doi.org/10.1182/blood-2015-04-641969

De Rasmo D, Signorile A, Roca E and Papa S (2009). cAMP response element-binding protein (CREB) is imported into mitochondria and promotes protein synthesis. FEBS J. 276: 4325-4333. http://dx.doi.org/10.1111/j.17424658.2009.07133.x

Dworkin S, Malaterre J, Hollande F, Darcy PK, et al. (2009). cAMP response element binding protein is required for mouse neural progenitor cell survival and expansion. Stem Cells 27: 1347-1357. http://dx.doi.org/10.1002/stem.56

Ferron L, Ruchon Y, Renaud JF and Capuano V (2011). T-type $\mathrm{Ca}^{2}+$ signalling regulates aldosterone-induced CREB activation and cell death through PP2A activation in neonatal cardiomyocytes. Cardiovasc. Res. 90: 105-112. http:// dx.doi.org $/ 10.1093 / \mathrm{cvr} / \mathrm{cvq} 379$

Han X, Zhao X, Lu M, Liu F, et al. (2013). Electroacupuncture ameliorates learning and memory via activation of the CREB signaling pathway in the hippocampus to attenuate apoptosis after cerebral hypoperfusion. Evid. Based Complement. Alternat. Med. 2013: 156489. http://dx.doi.org/10.1155/2013/156489

Hengartner MO (2000). The biochemistry of apoptosis. Nature 407: 770-776. http://dx.doi.org/10.1038/35037710

Horton WE, Jr., Bennion P and Yang L (2006). Cellular, molecular, and matrix changes in cartilage during aging and osteoarthritis. J. Musculoskelet. Neuronal Interact. 6: 379-381.

Kroemer G, Zamzami N and Susin SA (1997). Mitochondrial control of apoptosis. Immunol. Today 18: 44-51. http:// dx.doi.org/10.1016/S0167-5699(97)80014-X

Lane RS, Fu Y, Matsuzaki S, Kinter M, et al. (2015). Mitochondrial respiration and redox coupling in articular chondrocytes. Arthritis Res. Ther. 17: 54. http://dx.doi.org/10.1186/s13075-015-0566-9

Matsuo N, Tanaka S, Gordon MK, Koch M, et al. (2006). CREB-AP1 protein complexes regulate transcription of the collagen XXIV gene (Col24a1) in osteoblasts. J. Biol. Chem. 281: 5445-5452. http://dx.doi.org/10.1074/jbc. $\underline{\text { M509923200 }}$

Meng FJ, Jiao SM and Yu B (2012). Picroside II protects cardiomyocytes from hypoxia/reoxygenation-induced apoptosis by activating the PI3K/Akt and CREB pathways. Int. J. Mol. Med. 30: 263-270.

Milner PI, Wilkins RJ and Gibson JS (2007). The role of mitochondrial reactive oxygen species in pH regulation in 
articular chondrocytes. Osteoarthritis Cartilage 15: 735-742. http://dx.doi.org/10.1016/j.joca.2007.01.008

Misra MK, Sarwat M, Bhakuni P, Tuteja R, et al. (2009). Oxidative stress and ischemic myocardial syndromes. Med. Sci. Monit. 15: RA209-RA219.

Naqvi S, Martin KJ and Arthur JS (2014). CREB phosphorylation at Ser133 regulates transcription via distinct mechanisms downstream of cAMP and MAPK signalling. Biochem. J. 458: 469-479. http://dx.doi.org/10.1042/BJ20131115

Reed JC (1997). Cytochrome c: can't live with it - can't live without it. Cell 91: 559-562. http://dx.doi.org/10.1016/S0092$\underline{8674(00) 80442-0}$

Sato M, Bagchi D, Tosaki A and Das DK (2001). Grape seed proanthocyanidin reduces cardiomyocyte apoptosis by inhibiting ischemia/reperfusion-induced activation of JNK-1 and C-JUN. Free Radic. Biol. Med. 31: 729-737. http:// dx.doi.org/10.1016/S0891-5849(01)00626-8

Sun YQ, Zheng S, Yu J, Yan K, et al. (2013). Effect of total disc replacement on atypical symptoms associated with cervical spondylosis. Eur. Spine J. 22: 1553-1557. http://dx.doi.org/10.1007/s00586-013-2785-6

Triantafillou KM, Lauerman W and Kalantar SB (2012). Degenerative disease of the cervical spine and its relationship to athletes. Clin. Sports Med. 31: 509-520. http://dx.doi.org/10.1016/j.csm.2012.03.009

Vanharanta S (2015). A hypoxic ticket to the bone metastatic niche. Breast Cancer Res. 17: 122. http://dx.doi.org/10.1186/ s13058-015-0635-7

Vivanco I and Sawyers CL (2002). The phosphatidylinositol 3-Kinase AKT pathway in human cancer. Nat. Rev. Cancer 2: 489-501. http://dx.doi.org/10.1038/nrc839

Wang P, Huang S, Wang F, Ren Y, et al. (2013). Cyclic AMP-response element regulated cell cycle arrests in cancer cells. PLoS One 8: e65661. http://dx.doi.org/10.1371/journal.pone.0065661

White R and Gibson JS (2010a). The effect of oxygen tension on calcium homeostasis in bovine articular chondrocytes. $J$. Orthop. Surg. 5: 27. http://dx.doi.org/10.1186/1749-799X-5-27

White R and Gibson JS (2010b). The effect of oxygen tension on calcium homeostasis in bovine articular chondrocytes. $J$. Orthop. Surg. 5: 27. http://dx.doi.org/10.1186/1749-799X-5-27 